\title{
Hypersomnia in Mood Disorders: a Rapidly Changing Landscape
}

\author{
David T. Plante ${ }^{1,2}$
}

Published online: 17 April 2015

(C) Springer International Publishing AG 2015

\begin{abstract}
Hypersomnia is commonly comorbid with depressive illness and is associated with treatment resistance, symptomatic relapse, and functional impairment. This review highlights recent changes in nosological classifications of hypersomnia. In addition, emergent findings regarding the neurobiologic underpinnings, assessment, and treatment of hypersomnia in mood disorders are reviewed, as well as the effects of hypersomnolence on illness course. Future strategies for research are proposed that may elucidate the causes of hypersomnia in mood disorders and lead to the development of improved diagnostic and therapeutic strategies.
\end{abstract}

Keywords Hypersomnia $\cdot$ Nosology $\cdot$ Sleepiness $\cdot$ Mood disorders $\cdot$ Depression $\cdot$ Arousal

\section{Introduction}

Mood disorders, such as unipolar major depressive disorder (MDD) and bipolar disorder, are significant global public health problems [1]. Hypersomnia, defined as excessive daytime sleepiness (EDS) and/or total sleep time, commonly occurs in patients with mood disorders and is associated with

This article is part of the Topical Collection on Sleep and Psychological Disorders

David T. Plante

dplante@wisc.edu

1 Department of Psychiatry, University of Wisconsin School of Medicine and Public Health, Madison, WI, USA

2 Wisconsin Sleep, 6001 Research Park Blvd., Madison, WI 53719, USA treatment resistance, symptomatic relapse, increased risk of suicide, and functional impairment [2-7]. However, there has generally been scant research conducted to determine the underlying neurobiology of hypersomnia in mood disorders, clarify its effects on illness trajectory, develop objective means to assess its severity, and tailor pharmacologic therapies.

Building on prior reviews that have thoughtfully considered this complex topic $[4,8]$, this report will provide an updated synopsis of the recent literature on hypersomnia in mood disorders. In so doing, it will critically evaluate recent advances, highlight promising areas upon which research may build, and emphasize strategies for future investigation that may enhance our understanding of this important symptom in mood disorders.

\section{Shifting Nosology}

In the absence of a clear etiology or pathogenesis for hypersomnia occurring in mood disorders, classification is based predominantly on symptoms, with objective measures used as supporting evidence for the delineation of diagnoses among similar disorders. The two most commonly used nosological systems for the assessment and diagnosis of hypersomnia in depressive illness are the International Classification of Sleep Disorders (ICSD) and the Diagnostic and Statistical Manual (DSM). Both have recently been updated, to their third (ICSD-3) and fifth (DSM-5) respective editions, with several important changes made to diagnostic criteria in both manuals $[9,10]$.

In the ICSD-3, the primary nomenclature has transitioned to Hypersomnia Associated with a Psychiatric Disorder [10] from Hypersomnia Not Due to Substance or Known Physiological Condition in the ICSD-2 [11]. However, both terms (as well as others) are considered alternate names across versions. 
Also, the ICSD-2 required a complaint of EDS or excessive sleep; however, in the ICSD-3, a report of irrepressible need to sleep or daytime lapses into sleep are required, with a complaint of excessive total sleep time omitted from the primary diagnostic criteria and moved instead to the descriptive essential features. Additionally, the ICSD-3 now explicitly requires that the complaint occurs for at least 3 months, where the ICSD-2 previously did not have a duration criterion. Finally, the ICSD-2 required the following two specific objective criteria be met: reduced sleep efficiency and increased frequency and duration of awakenings on polysomnography, as well as variable, often normal, mean sleep latencies on the MSLT. These polysomnographic requirements have been removed from the diagnostic criteria in the ICSD-3.

The disorder that is most difficult to distinguish from Hypersomnia Associated with a Psychiatric Disorder is Idiopathic Hypersomnia (IH), particularly since $15-25 \%$ of patients with IH report depressive symptoms [8]. Central to the distinction between these disorders in the ICSD-3 are multiple sleep latency test (MSLT) findings. The ICSD-3 maintains the ICSD-2 standard that MSLT findings are usually normal in hypersomnia associated with a mood disorder, while the mean sleep latency in IH is $\leq 8 \mathrm{~min}$. However, the ICSD-3 now also allows for the diagnosis of IH if total 24-h sleep time is $\geq 11 \mathrm{~h}$ (performed after correction for chronic sleep deprivation), confirmed by continuous polysomnographic recording or wrist actigraphy in association with a sleep diary (averaged over at least 7 days with unrestricted sleep). This objective sleep duration criterion subsumes the prior ICSD-2 delineation between IH with and without long sleep time, largely due to insufficient evidence of validity in segregating these subtypes. However, the ICSD-3 does note that occasionally patients fulfilling other subjective criteria for IH may not fulfill either of these objective requirements for the disorder and that clinical judgment should be used in deciding if these patients should be considered to have $\mathrm{IH}$, further complicating the nosologic distinction between $\mathrm{IH}$ with comorbid depressive symptoms and hypersomnia associated with a mood disorder.

There have also been major revisions, both technical and conceptual, to hypersomnia in the DSM-5 compared to DSMIV $[9,12]$. First, Hypersomnolence Disorder in the DSM-5 has replaced Primary Hypersomnia in the DSM-IV. This diagnosis requires self-reported excessive sleepiness despite a main sleep period lasting at least $7 \mathrm{~h}$, with at least one of the following symptoms: recurrent periods of sleep or lapses into sleep within the same day, a prolonged main sleep episode of more than $9 \mathrm{~h}$ per day that is non-restorative, or difficulty being fully awake after abrupt awakenings [9]. The duration of symptoms must be at least 3 months (similar to ICSD-3), must cause significant distress or impairment, not be due to some other sleep disorder or exogenous substance, and coexisting mental and medical disorders cannot adequately explain the predominant complaint of hypersomnolence.
These criteria are very similar to those previously proposed by Ohayon and colleagues [13•] and more clearly delineate symptoms as compared to DSM-IV Primary Hypersomnia. It is noteworthy that the DSM-5 diagnosis of Hypersomnolence Disorder in many respects subsumes the ICSD-3 diagnoses of Idiopathic Hypersomnia and Hypersomnia Associated with a Psychiatric Disorder. Unlike the ICSD-3, MSLT findings are not part of the diagnostic criteria, and as described in the DSM-5, mean sleep latency on MSLT is typically less than $10 \mathrm{~min}$ and frequently $8 \mathrm{~min}$ or less in Hypersomnolence Disorder.

The DSM-5 marks a shift to a nosology that considers sleep disorders to be comorbid with, rather than secondary to, psychiatric disorders. The DSM-5 notes that many individuals with Hypersomnolence Disorder have symptoms of depression that may meet criteria for a depressive disorder and that Hypersomnolence Disorder can be diagnosed in the presence of another current or past mental disorder [9]. Notably, the DSM-IV did allow for diagnosis of both Primary Hypersomnia and another current or past mental disorder if the hypersomnia and psychiatric disorder were deemed to have an independent course [12]. However, given the bidirectional relationships between sleep and mood disorders [14], the DSM-5 has left distinctions drawn by course of illness more ambiguous, as frequently occurs in clinical situations. Further complicating nosologic distinctions are a lack of clear and consistent definitions of hypersomnia as a diagnostic feature of specific mood disorder subtypes in DSM-5. As a diagnostic criterion of MDD, hypersomnia is described as prolonged sleep episodes at night or increased daytime sleep [9]. However, hypersomnia occurring in the context of atypical depression is more clearly defined and requires either an extended period of nighttime sleep or daytime napping that totals $10 \mathrm{~h}$ of sleep per day (or at least $2 \mathrm{~h}$ more than when not depressed) [9]. The lack of parallelism in definitions of hypersomnia within different sections of the DSM-5 underscores the complexities faced by both clinicians and researchers in the study of hypersomnia in mood disorders.

The need for clear definitions of hypersomnia for diagnostic and research purposes has been demonstrated by a recent epidemiologic study by Ohayon and Roberts [15•]. In this investigation, the authors examined the association between oversleeping and overeating (i.e., reversed neurovegetative symptoms) in persons experiencing a major depressive episode in a large cross-sectional study. Notably, when hypersomnia was defined as self-reported total sleep time of $\geq 10 \mathrm{~h}$ per day or at least $2 \mathrm{~h}$ longer than during normothymic periods (similar to definitions used for atypical depression in DSM-5), there was no association with overeating [15•]. However, there was an association between a less defined hypersomnia definition, "getting too much sleep," and hyperphagia [15•]. These findings underscore the need to use strict definitions in defining hypersomnia in mood disorders 
research and challenge the underlying validity of atypical depression as a diagnostic construct, since factor analysis also failed to identify a clear element grouping the symptoms of atypical depression [15•].

\section{Impact on Hypersomnia on the Natural History of Mood Disorders}

When examining the role hypersomnia plays on the natural history of mood disorders, it is thus paramount to consider what facets of hypersomnia (i.e., excessive sleep time and/or sleepiness) are considered in a given investigation, as well as other factors that might be associated with impairment. Using population-based data, Ohayon and colleagues recently estimated that $8.4 \%$ of the population self-reports sleeping $\geq 9 \mathrm{~h}$ per night, but that only $1.6 \%$ experiences excessive sleep time and resultant distress or impairment in functioning, with the likelihood of this combination 3- to12-fold higher among persons with a mood disorder [16•]. However, the impact of excessive sleep time alone on psychosocial functioning may be relatively small compared to other factors, as suggested by Fried and Nesse [17], who examined the relative contributions of individual depressive symptoms to impairment in outpatients participating in the Sequenced Treatment Alternatives to Relieve Depression (STAR*D) trial. In this study, excessive sleep time had lower relative impact on psychosocial functioning than other factors; however, it is noteworthy that fatigue played a major role in impairment and that excessive sleepiness was not considered as a component in analyses [17]. In addition, the role of comorbid insomnia with excessive sleep time may have additive effects, as recently demonstrated by Soehner and colleagues [18•]. Using data from the National Comorbidity Survey Replication (NCS-R), these authors found that patients with difficulty initiating and/or maintaining sleep, in combination with self-reported excessive sleep time $\geq 10 \mathrm{~h}$, had more severe depressive episodes, comorbidities, and functional impairment than other depressive groups [18•]. However, the role of excessive sleepiness and cooccurring insomnia could not be evaluated based on limitations of the available data [18•].

Fernandez-Mendoza and colleagues recently examined polysomnographic factors at baseline that predicted incident EDS in the Penn State Adult Cohort study, after a mean 7.5-year follow-up period [19•]. Notably, they found significant interactions between depression and sleep architecture on incident sleepiness, such that incident EDS in depressed persons was associated with sleep disturbance (e.g., reduced sleep efficiency and longer sleep latency), which was opposite to factors associated with incident EDS among non-depressed individuals (e.g., short sleep onset latency and higher sleep efficiency) [19•].
Additional recent research has highlighted the varied importance of different components of hypersomnia on the clinical trajectory of patients with mood disorders. Kaplan and colleagues evaluated a sample of 159 bipolar patients (euthymic at study entry) at baseline and at 7-month followup [20]. Factor analysis demonstrated two separate subtypes of hypersomnia (long sleep and excessive sleepiness) that were not correlated. Among long sleepers, there was a discrepancy between total sleep time assessed by sleep diaries and actigraphy ( 8.80 vs. $7.69 \mathrm{~h}$ ), suggesting discordance between subjective and objective measures of sleep duration. The authors suggest that long sleep in these participants thus reflected increased time in bed rather than sleep duration [20•]. In addition, excessive sleepiness, but not long sleep, predicted relapse to mania/hypomania in this sample, suggesting this facet of hypersomnia may play a particularly important role in the course of the disorder [20•]. However, the connections between constellations of symptoms and clinical course must be bridged by simultaneous efforts to clarify the neurobiologic processes that underlie them, if optimal therapeutics and preventative strategies are to be developed.

\section{Neurobiology of Hypersomnia in Mood Disorders}

There have been several recent studies that may provide insights into the underlying neurobiology responsible for hypersomnia in mood disorders. Using data from a large twin registry, Watson and colleagues [21•] examined the magnitude of genetic and environmental influences on self-reported sleep duration and depressive symptoms, as well as how sleep duration moderated genetic influence on depression. Individuals with both short $(<7 \mathrm{~h})$ and long $(\geq 9 \mathrm{~h}$ ) sleep duration reported significantly more depressive symptoms than normal sleepers [21•]. Moreover, genetic influences on depressive symptoms were more pronounced among those with both short and long sleep duration, with heritability of depressive symptoms increasing nearly 2 -fold among those sleeping $10 \mathrm{~h} /$ night (from $27 \%$ for normal duration sleepers to $49 \%$ among long sleepers) [21•]. These results are congruent with previous twin studies that have demonstrated significant genetic correlation between depressive symptoms and daytime sleepiness as measured by the Epworth Sleepiness Scale (ESS) [22]. Although neither investigation was able to identify specific pleiotropic genes responsible for these observed effects, potential candidates include those related to circadian rhythms, cellular metabolism, and monoamine activity, based on several studies that have connected polymorphisms within these gene classes with sleep duration [23-28].

The majority of genetic polymorphisms identified that affect sleep duration have been associated with short, rather than excessive sleep times. However, prior work in two independent populations demonstrated associations within the 
CLOCK gene intronic region (rs12649507/rs11932595) with long sleep, defined as sleep duration $>8.5 \mathrm{~h}$ [29]. However, this association was confirmed only in one of seven cohorts included in a recent genome-wide association study, raising questions about the importance of this gene in the pathophysiology of hypersomnolence [25].

Other genes that may be particularly pertinent to hypersomnia in mood disorders are those related to monoamine oxidase A (MAO-A) activity. Specifically, a functional variable-number tandem repeat (VNTR) in the promoter region of the MAO-A gene has been a focus of neuropsychiatric research across multiple disorders, including mood disorders [30]. The number of repeats in the VNTR allele affects the transcription of MAO-A, with 3.5 or 4 copies of the repeat more efficiently transcribed than those with 3 or 5 copies [31]. Recently, an association between MAO-A VNTR and daytime sleepiness (as measured by the ESS score) has been demonstrated in healthy persons [32]. In this study, alleles associated with more efficient transcription were associated with increased sleepiness. Since serotonin, norepinephrine, and dopamine are all key constituents of the ascending arousal system [33], increased MAO-A activity would theoretically decrease the levels of these monoamines, thus resulting in sleepiness from diminished arousal. However, activity of MAO-A as assayed by cerebrospinal fluid monoamine metabolites has not consistently demonstrated expected correlations between VNTR genotype and MAO-A activity, including prior work in atypical depression [34-36]. This is likely due to several complex factors that influence the transcription of the MAO-A gene, including epigenetic and hormonal regulation [37].

The role of glucocorticoids in the transcription and subsequent activity of MAO-A may be particularly pertinent to hypersomnia in mood disorders. Le Dantec and colleagues [38•] have recently described hypersomnia in a murine model of depression using chronic corticosterone administration. Specifically, these animals demonstrated increased sleep time, intrusion of NREM sleep into the dark (active) phase, and decreases in sleep continuity during the light (inactive) phase [38•]. Notably, glucocorticoid administration increases the transcription rate and catalytic activity of MAO-A in human cell lines, connecting changes in the hypothalamic-pituitary-adrenal (HPA) axis that occur in the high stress state of depression, with upregulation of MAO-A [39, 40]. In the case of hypersomnia in mood disorders, it is also noteworthy that recent evidence utilizing positron emission tomography has also demonstrated elevated MAO-A binding in the prefrontal and anterior cingulate cortices in patients with reversed neurovegetative symptoms (i.e., hypersomnia and hyperphagia) [41]. However, because patients with atypical depression demonstrate lower diurnal cortisol slopes with normal serum cortisol levels [42], connections between hypersomnia in mood disorders and the HPA axis are speculative, and if present, may be due to negative feedback and/or altered sensitivity of the system.

Despite emerging lines of research, the underlying neurobiology of hypersomnia in mood disorders remains to be elucidated. A non-exhaustive list of other possible biological mechanisms that may underlie hypersomnia in mood disorders includes alterations in sleep homeostasis, a slowed circadian pacemaker, and/or increased inflammatory cytokines [43-45]. At this juncture, there is not definitive evidence for any specific pathologic process that is responsible for hypersomnolence in depression. Complicating research in this area are several factors such as inconsistent definitions/criteria used to define hypersomnolence, a significant probability that hypersomnia in mood disorders is due to several interrelated factors, and inadequate objective measures to assess hypersomnia in depression. In the absence of a defined pathophysiology, the treatment of hypersomnolence in mood disorders is thus based primarily on symptomatic response to medications that were initially developed for other purposes.

\section{Treatments for Hypersomnia in Mood Disorders}

There are currently no pharmacologic agents that have been approved to treat hypersomnia in mood disorders. However, several agents have been examined for their effects on sleepiness in depression, often as a secondary endpoint in antidepressant efficacy studies or as an augmenting agent in patients with residual hypersomnia symptoms.

Non-stimulant wake-promoting agents, modafinil and armodafinil, which are approved for the treatment of excessive sleepiness associated with narcolepsy, obstructive sleep apnea, and shift work [46, 47], have received significant attention as a possible treatment for hypersomnolence in mood disorders. A recent meta-analysis examined the efficacy of these agents as adjunctive therapy with standard treatment (antidepressants and/or mood stabilizers) in patients with unipolar or bipolar depression [48]. Pooled results from five studies included in a secondary endpoint analysis suggested these medications did not improve sleepiness [48]. However, in a subset of three studies that reported data at 1 week, modafinil did improve subjective EDS [48]. These meta-analytic findings must be interpreted with caution, however, because only two of the five studies in the pooled analysis required an ESS score $\geq 10$ (indicative of a significant level of sleepiness) as an inclusion criterion [48], suggesting failure to see changes in ESS scores may have been due to a floor effect in some studies. Moreover, one study that was included in analyses utilized the change in item \#4 on the Inventory of Depressive Symptomatology as its hypersomnia outcome measure [49], while the other studies utilized change in the ESS [50-53]. Because the former is a measure of total sleep time, but the latter a measure of tendency to doze in various situations, it is not 
clear whether results would have differed if only data comparing similar components of hypersomnia were considered.

Beyond non-stimulant wake-promoting agents, lisdexamphetamine, a prodrug of dextroamphetamine, has also been recently examined as an adjunctive treatment of bipolar depression in a double-blind randomized controlled trial (RCT), with change in ESS score included as a secondary endpoint [54]. Although the sample size was limited $(n=25)$ due to premature study termination by the funding sponsor, significant improvements in ESS scores were observed at 8 weeks of treatment [54]. However, the use of stimulant medications in patients with bipolar disorder is controversial, and potential risks and benefits must be carefully considered, particularly in light of the concern for treatment-emergent affective switches that can be associated with this class of medications $[55,56]$.

One of the inherent difficulties in evaluating the role of stimulants and wake-promoting agents as adjunctive therapies for hypersomnia in mood disorders is the possibility that sleepiness is related to an antidepressant treatment, rather than the disorder itself. Hypersomnia is frequently a residual symptom that remains despite remission of a major depressive episode treated pharmacologically $[2,3]$. Moreover, recent data from the STAR*D trial suggest that among MDD patients who do not remit despite citalopram therapy, hypersomnia, unlike other symptoms, does not improve over the course of treatment [57]. Meta-analytic data has also suggested that selective serotonin reuptake inhibitors (SSRIs), the most common agents used to treat depression, are associated with significantly higher rates of somnolence as a side effect compared to placebo and bupropion, a norepinephrine/dopamine reuptake inhibitor [58]. Pooled data from six double-blind RCTs, which utilized the sum of items \#22 (early bedtime/ falling asleep earlier than usual), \#23 (oversleeping/waking up later than usual in the morning), and \#24 (daytime napping/going to bed early) on the Hamilton Depression Rating Scale (HDRS) as the measure of hypersomnia, have previously suggested that bupropion results in greater resolution of sleepiness in patients treated for depression than SSRIs and placebo [59]. More recently, data from these same studies has been re-analyzed by examining only participants using bupropion at doses $\leq 300 \mathrm{mg}$ daily (commensurate with European Union approved dose) and in those achieving remission from depression [60]. Bupropion was associated with similar rates of depression remission as SSRIs, but fewer bupropiontreated remitters had residual symptoms of sleepiness, suggesting bupropion may be of greater value than SSRIs in the management of hypersomnolence comorbid with mood disorders [60].

Although the majority of studies have examined the role of arousal-promoting agents in the treatment of sleepiness associated with mood disorders, another group of investigators evaluated the effects of the sedating antidepressant, mirtazapine, on measures of EDS in patients with MDD. Shen and colleagues [61] conducted an open-label treatment study using mirtazapine, assessing changes to both subjective (ESS and Stanford Sleepiness Scale; SSS) and objective (mean sleep latency on MSLT) sleepiness in patients with MDD. The authors found both subjective and objective measures of sleepiness demonstrated a biphasic response to mirtazapine, with acute worsening early in the course of therapy but significant improvement relative to baseline by the end of treatment [61]. However, applicability of this therapy in the treatment of hypersomnolent mood-disordered patients must be tempered by the fact that hypersomnia was not an inclusion/exclusion criterion and all study participants had some degree of insomnia [62], which, when combined with a relatively low mean sleep latency on baseline MSLT (7.8 $\pm 5.0 \mathrm{~min}$ ) [61], suggests this therapy may be better tailored to patients with cooccurring insomnia and excessive sleepiness. Notably, mirtazapine enhanced slow wave sleep in these patients, but did not significantly alter sleep efficiency or total sleep time [62]. Mirtazapine has activity as a 5HT-2A antagonist, which is likely the mechanism through which it enhances slow wave activity (SWA) in both healthy and mood-disordered persons [63-65]. Thus, improvements in hypersomnia through enhancement of SWA would be congruent with hypothesized deficits in sleep homeostatic function in hypersomnolent depressive individuals [43], and although speculative, suggest that the enhancement of SWA may be a fruitful target of treatment in future research.

\section{Measuring Hypersomnia in Mood Disorders}

Perhaps the greatest challenge for research on hypersomnolence in mood disorders is the lack of objective measures that can be used for diagnostic purposes and measuring treatment outcomes. The MSLT, widely considered the gold standard of objective sleepiness, has not demonstrated consistent findings in mood-disordered patients with hypersomnia [66-68]. Recently, Kofmel and colleagues [69•] compared several objective measures of hypersomnia in patients with mood disorders (depression or dysthymia) who complained of excessive sleepiness (ESS $\geq 10$ ), with patients with narcolepsy and obstructive sleep apnea. Interestingly, these authors found a high percentage of these mood-disordered patients (12/19) demonstrated a mean sleep latency on MSLT of $<8 \mathrm{~min}$, consistent with a pathologic level of sleepiness (Kofmel 2014). This proportion is higher than previous investigations that have reported roughly $21-22 \%$ of hypersomolent mooddisordered patients demonstrate pathologic sleep latencies on the MSLT $[68,70]$. These findings highlight the fact that the MSLT is likely not particularly useful to either rule in or rule out hypersomnia in mood disorders, as a modest percentage of 
these patients will demonstrate a pathologic sleep latency on MSLT; however, the majority will not.

Limitations of the MSLT highlight the need for other objective measures of hypersomnia in mood disorders. The MSLT, which measures the ability to fall asleep, does not capture all the facets encompassed by hypersomnia. For example, it does not measure the ability to stay awake, which may be assessed with the maintenance of wakefulness test (MWT) [71]. Patients with hypersomnolence often demonstrate discordant results on the MSLT and MWT, including patients with psychiatric disorders [70]. Moreover, the ESS, MSLT, and MWT are generally not well correlated with one another, complicating their interpretation and use in patients with mood disorders [71].

Two aspects of hypersomnia not considered in traditional measures of sleepiness that might help distinguish hypersomnolent patients with psychiatric disorders from other disorders of excessive sleepiness are sleep inertia and time-ontask-related performance. Peter-Derex and colleagues [72•], recently evaluated the use of auditory evoked potentials during a forced-awakening nap protocol as a measure of excess sleep inertia (the inability to attain full alertness following awakening from sleep). They found that P300 latencies were delayed during forced awakenings in patients with sleep apnea, narcolepsy, and idiopathic hypersomnia, but not psychiatric patients who complained of excessive sleepiness, suggesting this measure might help distinguish psychiatric from neurological forms of hypersomnia [72•]. Kofmel and colleagues [69•] recently demonstrated hypersomnolent mooddisordered patients did more poorly relative to other hypersomnolent patient groups on the Steer Clear task, a monotonous 30 -min vigilance task in which patients avoid intermittent obstacles [73]. These results suggest that objective deficits associated with hypersomnia in mood disorders may be related to extended time-on-task, attentional issues, and/or motivational factors.

\section{Conclusion and Future Directions}

Hypersomnia clearly plays a key role in the course of mood disorders. However, in the absence of established neurobiologic mechanisms that underlie this symptom, the chasm between basic science and effective clinical treatments remains quite wide. Refinement of our research methodologies is a vital issue if we hope to translate scientific discovery into clinical care. The heterogeneous nature of hypersomnia, which includes several aspects such as sleep propensity, ability to maintain wakefulness, excessive sleep inertia, increased total sleep time, and impaired attention/motivation, which often co-occur, but may frequently be discordant between subjective and objective measures, as well as within patient groups, is a crucial issue to address. Prior research has used varied definitions of hypersomnia, which frequently fail to capture the multifaceted nature of the complaint, complicating further the generalizability of such studies.

One strategy that may enhance future research is the use of specific diagnostic criteria for hypersomnolence disorder, such as those proposed by Ohayon and colleagues [13•] that are reflected in the current DSM-5 [9]. Such diagnostic criteria are based on large-scale epidemiologic data, and thus more apt to be useful and valid for both clinical and research purposes than previous nosologies. In addition, these diagnostic schemas take into account the multi-dimensional aspects of hypersomnia, which may in turn enhance basic science research, particularly if individual facets of hypersomnolence (excessive total sleep time, irresistible need to sleep, etc.) can be mapped more carefully onto specific neurobiologic constructs.

In many ways, the challenges facing hypersomnia research are a microcosm of those faced by broader psychiatric research. Hypersomnia is experienced and/or observed in multiple medical, neurologic, and psychiatric disorders. Such sharp delineations between disciplines and disease categories, however, are frequently based more on historical convention and/or convenience than science, particularly among disorders of hypersomnolence for which the etiology and pathophysiology remains elusive. The unifying notion around which collaborative research should focus is that hypersomnia, and all of its dimensions, ultimately results from or is manifest by brain dysfunction.

In an effort to break down specific limitations inherent to diagnostic strategies based on clusters of signs and symptoms, the National Institute of Mental Health (NIMH) has developed the Research Domain Criteria (RDoC), which supports research to elucidate fundamental biobehavioral dimensions that cut across heterogeneous disorder categories [74, 75]. Within RDoC, hypersomnia research spans the core Domain of Arousal/Modulatory Systems. Under this schema, research in hypersomnia would utilize multiple scientific methods/ paradigms to examine different units of analysis (genetics, molecules, cells, brain circuits, physiology, behavior, and self-report) across multiple disorders, rather than solely within one specific diagnostic category. This new way to conceptualize neuropsychiatric investigation provides a potentially fertile environment in which novel transdiagnostic approaches and methods to study hypersomnia may thrive. However, progress in this sphere will likely depend upon collaborative and cognitive flexibility among researchers across multiple disciplines. As such, this challenge must be met with enthusiasm and a sense of solidarity that these paradigms of biomedical inquiry will enhance our understanding of the mind, brain, and behavior and ultimately translate into improved treatments for all patients who are afflicted with hypersomnolence. 


\section{Compliance with Ethics Guidelines}

Conflict of Interest David T. Plante has received grants from the National Institute of Mental Health, Brain and Behavior Research Foundation and American Sleep Medicine Foundation.

Human and Animal Rights and Informed Consent This article does not contain any studies with human or animal subjects performed by any of the authors.

\section{References}

Papers of particular interest, published recently, have been highlighted as:

- Of importance

1. Collins PY, Patel V, Joestl SS, et al. Grand challenges in global mental health. Nature. 2011;475(7354):27-30.

2. Worthington J, Fava M, Davidson K, Alpert J, Nierenberg AA, Rosenbaum JF. Patterns of improvement in depressive symptoms with fluoxetine treatment. Psychopharmacol Bull. 1995;31(2):2236.

3. Zimmerman M, McGlinchey JB, Posternak MA, Friedman M, Boerescu D, Attiullah N. Differences between minimally depressed patients who do and do not consider themselves to be in remission. J Clin Psychiatry. 2005;66(9):1134-8.

4. Kaplan KA, Harvey AG. Hypersomnia across mood disorders: a review and synthesis. Sleep Med Rev. 2009;13(4):275-85.

5. Kaplan KA, Gruber J, Eidelman P, Talbot LS, Harvey AG. Hypersomnia in inter-episode bipolar disorder: does it have prognostic significance? J Affect Disord. 2011;132(3):438-44.

6. Goldstein TR, Bridge JA, Brent DA. Sleep disturbance preceding completed suicide in adolescents. J Consult Clin Psychol. 2008;76(1):84-91.

7. Fitzgerald CT, Messias E, Buysse DJ. Teen sleep and suicidality: results from the youth risk behavior surveys of 2007 and 2009. J Clin Sleep Med. 2011;7(4):351-6.

8. Dauvilliers Y, Lopez R, Ohayon M, Bayard S. Hypersomnia and depressive symptoms: methodological and clinical aspects. BMC Med. 2013;11:78.

9. American Psychiatric Association. Diagnostic and statistical manual of mental disorders. 5th ed. Arlington, VA: American Psychiatric Association; 2013.

10. American Academy of Sleep Medicine. International classification of sleep disorders. 3rd ed. Darien, IL: American Academy of Sleep Medicine; 2014.

11. American Academy of Sleep Medicine: International classification of sleep disorders: diagnostic and coding manual. 2nd ed. Darien, IL: American Academy of Sleep Medicine; 2005.

12. American Psychiatric Association. Diagnostic and statistical manual of mental disorders. 4th ed., text revision. Washington, DC: American Psychiatric Association; 2000.

13. Ohayon MM, Dauvilliers Y, Reynolds 3rd CF. Operational definitions and algorithms for excessive sleepiness in the general population: implications for DSM-5 nosology. Arch Gen Psychiatry. 2012;69(1):71-9. Large epidemiologic study that examined the prevalence of excessive sleepiness and hypersomnolence disorder in the U.S. population.

14. Franzen PL, Buysse DJ. Sleep disturbances and depression: risk relationships for subsequent depression and therapeutic implications. Dialogues Clin Neurosci. 2008;10(4):473-81.
15. Ohayon MM, Roberts LW. Challenging the validity of the association between oversleeping and overeating in atypical depression. J Psychosom Res. 2015;78(1):52-7. Cross-sectional epidemiologic study examining the prevalence of depression and associated symptoms that showed no association between hypersomnia and other symptoms of atypical depression when hypersomnia was strictly defined.

16. Ohayon MM, Reynolds CF, Dauvilliers Y. Excessive sleep duration and quality of life. Ann Neurol. 2013;73(6):785-94. Large epidemiologic study that determined the population prevalence of sleeping greater than 9 hours per night with associated distress/impairment (1.6\%), with the likelihood of these symptoms 3-12-fold higher among individuals with a mood disorder.

17. Fried EI, Nesse RM. The impact of individual depressive symptoms on impairment of psychosocial functioning. PLoS ONE. 2014;9(2): e90311.

18. Soehner AM, Kaplan KA, Harvey AG. Prevalence and clinical correlates of co-occurring insomnia and hypersomnia symptoms in depression. J Affect Disord. 2014;167:93-7. Analysis of data from the National Comorbidity Survey Replication demonstrating co-occurring excessive sleep time and insomnia were associated with more severe major depressive episode.

19. Fernandez-Mendoza J, N A, Kritikou I, L S, Liao D, Bixler EO: Natural History of Excessive Daytime Sleepiness: Role of Obesity, Weight Loss, Depression, and Sleep Propensity. Sleep. 2015;38(3): 351-60. Using longitudinal data from the Penn State Adult Cohort, demonstrated sleep disturbance predicted incident daytime sleepiness in depressed individuals, which was opposite factors associated with incident sleepiness among nondepressed individuals.

20. Kaplan KA, McGlinchey EL, Soehner A, et al.: Hypersomnia subtypes, sleep and relapse in bipolar disorder. Psychol Med. 2014:113. In Press. Longitudinal analysis of a community sample of patients with bipolar disorder that described two separate (and uncorrelated) subtypes of hypersomnia, long sleep and excessive sleepiness, of which only the latter was associated with relapse to mania/hypomania.

21. Watson NF, Harden KP, Buchwald D, et al. Sleep duration and depressive symptoms: a gene-environment interaction. Sleep. 2014;37(2): 351-8. Using data from a large twin registry, demonstrated increased genetic influence on depressive symptoms among those with both short and long sleep duration.

22. Lessov-Schlaggar CN, Bliwise DL, Krasnow RE, Swan GE, Reed $\mathrm{T}$. Genetic association of daytime sleepiness and depressive symptoms in elderly men. Sleep. 2008;31(8):1111-7.

23. He Y, Jones CR, Fujiki N, et al. The transcriptional repressor DEC2 regulates sleep length in mammals. Science. 2009;325(5942):866-70.

24. Partonen T, Treutlein J, Alpman A, et al. Three circadian clock genes Per2, Arntl, and Npas2 contribute to winter depression. Ann Med. 2007;39(3):229-38.

25. Allebrandt KV, Amin N, Müller-Myhsok B, et al. A K(ATP) channel gene effect on sleep duration: from genome-wide association studies to function in Drosophila. Mol Psychiatry. 2013;18(1):12232.

26. Parsons MJ, Lester KJ, Barclay NL, Nolan PM, Eley TC, Gregory AM. Replication of genome-wide association studies (GWAS) loci for sleep in the British G1219 cohort. Am J Med Genet B Neuropsychiatr Genet. 2013;162B(5):431-8.

27. Benedetti F, Serretti A, Colombo C, et al. Influence of a functional polymorphism within the promoter of the serotonin transporter gene on the effects of total sleep deprivation in bipolar depression. Am J Psychiatry. 1999;156(9):1450-2.

28. Carskadon MA, Sharkey KM, Knopik VS, McGeary JE. Short sleep as an environmental exposure: a preliminary study associating 5-HTTLPR genotype to self-reported sleep duration and depressed mood in first-year university students. Sleep. 2012;35(6):791-6. 
29. Allebrandt KV, Teder-Laving M, Akyol M, et al. CLOCK gene variants associate with sleep duration in two independent populations. Biol Psychiatry. 2010;67(11):1040-7.

30. Fan M, Liu B, Jiang T, Jiang X, Zhao H, Zhang J. Meta-analysis of the association between the monoamine oxidase-A gene and mood disorders. Psychiatr Genet. 2010;20(1):1-7.

31. Sabol SZ, Hu S, Hamer D. A functional polymorphism in the monoamine oxidase A gene promoter. Hum Genet. 1998;103(3): 273-9.

32. Ojeda DA, Niño CL, López-León S, Camargo A, Adan A, Forero DA. A functional polymorphism in the promoter region of MAOA gene is associated with daytime sleepiness in healthy subjects. $\mathrm{J}$ Neurol Sci. 2014;337(1-2):176-9.

33. Saper CB, Scammell TE, Lu J. Hypothalamic regulation of sleep and circadian rhythms. Nature. 2005;437(7063):1257-63.

34. Aklillu E, Karlsson S, Zachrisson OO, Ozdemir V, Agren H. Association of MAOA gene functional promoter polymorphism with CSF dopamine turnover and atypical depression. Pharmacogenet Genomics. 2009;19(4):267-75.

35. Zalsman G, Huang YY, Harkavy-Friedman JM, Oquendo MA, Ellis SP, Mann JJ. Relationship of MAO-A promoter (u-VNTR) and COMT (V158M) gene polymorphisms to CSF monoamine metabolites levels in a psychiatric sample of Caucasians: a preliminary report. Am J Med Genet B Neuropsychiatr Genet. 2005;132B(1):100-3.

36. Ducci F, Newman TK, Funt S, Brown GL, Virkkunen M, Goldman D. A functional polymorphism in the MAOA gene promoter (MAOA-LPR) predicts central dopamine function and body mass index. Mol Psychiatry. 2006;11(9):858-66.

37. Shih JC, Wu JB, Chen K. Transcriptional regulation and multiple functions of MAO genes. J Neural Transm. 2011;118(7):979-86.

38. Le Dantec Y, Hache G, Guilloux JP, et al. NREM sleep hypersomnia and reduced sleep/wake continuity in a neuroendocrine mouse model of anxiety/depression based on chronic corticosterone administration. Neuroscience. 2014;274:357-68. Describes a neuroendocrine mouse model of depression in which corticosterone administration results in increased sleep time and intrusion of NREM sleep into the active phase of the animal.

39. Ou XM, Chen K, Shih JC. Glucocorticoid and androgen activation of monoamine oxidase A is regulated differently by R1 and Sp1. J Biol Chem. 2006;281(30):21512-25.

40. Gold PW. The organization of the stress system and its dysregulation in depressive illness. Mol Psychiatry. 2015;20(1):32-47.

41. Chiuccariello L, Houle S, Miler L, et al. Elevated monoamine oxidase a binding during major depressive episodes is associated with greater severity and reversed neurovegetative symptoms. Neuropsychopharmacology. 2014;39(4):973-80.

42. Lamers F, Vogelzangs N, Merikangas KR, de Jonge P, Beekman AT, Penninx BW. Evidence for a differential role of HPA-axis function, inflammation and metabolic syndrome in melancholic versus atypical depression. Mol Psychiatry. 2013;18(6):692-9.

43. Plante DT, Landsness EC, Peterson MJ, et al. Altered slow wave activity in major depressive disorder with hypersomnia: a high density EEG pilot study. Psychiatry Res. 2012;201(3):240-4.

44. Aeschbach D, Sher L, Postolache TT, Matthews JR, Jackson MA, Wehr TA. A longer biological night in long sleepers than in short sleepers. J Clin Endocrinol Metab. 2003;88(1):26-30.

45. Vgontzas AN, Papanicolaou DA, Bixler EO, Kales A, Tyson K, Chrousos GP. Elevation of plasma cytokines in disorders of excessive daytime sleepiness: role of sleep disturbance and obesity. J Clin Endocrinol Metab. 1997;82(5):1313-6.

46. Keating GM, Raffin MJ. Modafinil: a review of its use in excessive sleepiness associated with obstructive sleep apnoea/hypopnoea syndrome and shift work sleep disorder. CNS Drugs. 2005;19(9): 785-803.
47. Garnock-Jones KP, Dhillon S, Scott LJ. Armodafinil. CNS Drugs. 2009;23(9):793-803.

48. Goss AJ, Kaser M, Costafreda SG, Sahakian BJ, Fu CH. Modafinil augmentation therapy in unipolar and bipolar depression: a systematic review and meta-analysis of randomized controlled trials. J Clin Psychiatry. 2013;74(11):1101-7.

49. Calabrese JR, Ketter TA, Youakim JM, Tiller JM, Yang R, Frye MA. Adjunctive armodafinil for major depressive episodes associated with bipolar I disorder: a randomized, multicenter, doubleblind, placebo-controlled, proof-of-concept study. J Clin Psychiatry. 2010;71(10):1363-70.

50. DeBattista C, Doghramji K, Menza MA, Rosenthal MH, Fieve RR. Group MiDS: adjunct modafinil for the short-term treatment of fatigue and sleepiness in patients with major depressive disorder: a preliminary double-blind, placebo-controlled study. J Clin Psychiatry. 2003;64(9):1057-64.

51. Fava M, Thase ME, DeBattista C. A multicenter, placebocontrolled study of modafinil augmentation in partial responders to selective serotonin reuptake inhibitors with persistent fatigue and sleepiness. J Clin Psychiatry. 2005;66(1):85-93.

52. Dunlop BW, Crits-Christoph P, Evans DL, et al. Coadministration of modafinil and a selective serotonin reuptake inhibitor from the initiation of treatment of major depressive disorder with fatigue and sleepiness: a double-blind, placebo-controlled study. J Clin Psychopharmacol. 2007;27(6):614-9.

53. Frye MA, Grunze H, Suppes T, et al. A placebo-controlled evaluation of adjunctive modafinil in the treatment of bipolar depression. Am J Psychiatry. 2007;164(8):1242-9.

54. McElroy SL, Martens BE, Mori N, et al. Adjunctive lisdexamfetamine in bipolar depression: a preliminary randomized, placebo-controlled trial. Int Clin Psychopharmacol. 2015;30(1):613.

55. Grunze H. Stimulants for treating bipolar disorder: pro and con. Harv Rev Psychiatry. 2014;22(6):358-62.

56. Corp SA, Gitlin MJ, Altshuler LL. A review of the use of stimulants and stimulant alternatives in treating bipolar depression and major depressive disorder. J Clin Psychiatry. 2014;75(9):1010-8.

57. Sakurai $\mathrm{H}$, Uchida $\mathrm{H}$, Abe $\mathrm{T}$, et al. Trajectories of individual symptoms in remitters versus non-remitters with depression. J Affect Disord. 2013;151(2):506-13.

58. Thase ME, Haight BR, Richard N, et al. Remission rates following antidepressant therapy with bupropion or selective serotonin reuptake inhibitors: a meta-analysis of original data from 7 randomized controlled trials. J Clin Psychiatry. 2005;66(8):974-81.

59. Papakostas GI, Nutt DJ, Hallett LA, Tucker VL, Krishen A, Fava M. Resolution of sleepiness and fatigue in major depressive disorder: a comparison of bupropion and the selective serotonin reuptake inhibitors. Biol Psychiatry. 2006;60(12):1350-5.

60. Cooper JA, Tucker VL, Papakostas GI. Resolution of sleepiness and fatigue: a comparison of bupropion and selective serotonin reuptake inhibitors in subjects with major depressive disorder achieving remission at doses approved in the European Union. J Psychopharmacol. 2014;28(2):118-24.

61. Shen J, Hossain N, Streiner DL, et al. Excessive daytime sleepiness and fatigue in depressed patients and therapeutic response of a sedating antidepressant. J Affect Disord. 2011;134(1-3): 421-6.

62. Shen J, Chung SA, Kayumov L, et al. Polysomnographic and symptomatological analyses of major depressive disorder patients treated with mirtazapine. Can J Psychiatry. 2006;51(1):27-34.

63. Schmid DA, Wichniak A, Uhr M, et al. Changes of sleep architecture, spectral composition of sleep EEG, the nocturnal secretion of cortisol, ACTH, GH, prolactin, melatonin, ghrelin, and leptin, and the DEX-CRH test in depressed patients during treatment with mirtazapine. Neuropsychopharmacology. 2006;31(4):832-44. 
64. de Boer T. The effects of mirtazapine on central noradrenergic and serotonergic neurotransmission. Int Clin Psychopharmacol. 1995;10 (Suppl 4):19-23.

65. Dijk DJ. Slow-wave sleep deficiency and enhancement: implications for insomnia and its management. World J Biol Psychiatry. 2010;11 (Suppl 1):22-8.

66. Nofzinger EA, Thase ME, Reynolds 3rd CF, et al. Hypersomnia in bipolar depression: a comparison with narcolepsy using the multiple sleep latency test. Am J Psychiatry. 1991;148(9):1177-81.

67. Dolenc L, Besset A, Billiard M. Hypersomnia in association with dysthymia in comparison with idiopathic hypersomnia and normal controls. Pflugers Arch - Eur J Physiol. 1996;431(6 Suppl 2):R303-4.

68. Billiard M, Dolenc L, Aldaz C, Ondze B, Besset A. Hypersomnia associated with mood disorders: a new perspective. J Psychosom Res. 1994;38 Suppl 1:41-7.

69. Kofmel NC, Schmitt WJ, Hess CW, Gugger M, Mathis J. Sleepiness and performance is disproportionate in patients with non-organic hypersomnia in comparison to patients with narcolepsy and mild to moderate obstructive sleep apnoea. Neuropsychobiology. 2014;70(3):189-94. Compared objective measures of sleepiness and performance in patients with nonorganic hypersomnia, narcolepsy, and sleep apnea, demonstrating patients with hypersomnia associated with a psychiatric disorder had a disproportionately high error rate on the Steer Clear performance test.

70. Sangal RB, Thomas L, Mitler MM. Maintenance of wakefulness test and multiple sleep latency test. Measurement of different abilities in patients with sleep disorders. Chest. 1992;101(4):898-902.

71. Sullivan SS, Kushida CA. Multiple sleep latency test and maintenance of wakefulness test. Chest. 2008;134(4):854-61.

72. Peter-Derex L, Perrin F, Petitjean T, Garcia-Larrea L, Bastuji H. Discriminating neurological from psychiatric hypersomnia using the forced awakening test. Neurophysiol Clin. 2013;43(3):171-9. Using auditory evoked potentials during a forced awakening nap protocol, demonstrated patients with hypersomnia associated with a mood disorder did not have objectively measured excessive sleep inertia compared to other disorders of hypersomnolence.

73. Findley L, Unverzagt M, Guchu R, Fabrizio M, Buckner J, Suratt P. Vigilance and automobile accidents in patients with sleep apnea or narcolepsy. Chest. 1995;108(3):619-24.

74. Cuthbert BN, Insel TR. Toward the future of psychiatric diagnosis: the seven pillars of RDoC. BMC Med. 2013;11:126.

75. Insel TR. The NIMH Research Domain Criteria (RDoC) Project: precision medicine for psychiatry. Am J Psychiatry. 2014;171(4): 395-7. 